\title{
Semiotik
}

Band $37 \cdot$ Heft 1-2 (2015)

Seite 177-187

Stauffenburg Verlag Tübingen

\section{Die Struktur und Funktion von mimischen Emotikons in Deutschland und in China}

\author{
Yixin Wu und Christian Trautsch, Technische Universität Berlin
}

\begin{abstract}
Summary. In the last two decades emoticons have become one of the most efficient means for the expression of emotions in written texts. Especially popular are emoticons with the facial traits of laughing and smiling persons. The present article analyzes the results of a comparative study of Chinese and German emoticons with special consideration of the relation between signs and objects.
\end{abstract}

Zusammenfassung. In den letzten zwei Jahrzehnten haben sich Emotikons zu einem der effizientesten Mittel für den Ausdruck von Emotionen in geschriebenen Texten entwickelt. Besonders beliebt sind Emotikons, in denen lachende und lächelnde Gesichter dargestellt werden. Der vorliegende Beitrag analysiert die Ergebnisse einer vergleichenden Studie von chinesischen und deutschen Emotikons unter besonderer Berücksichtigung der Beziehung zwischen Zeichen und Objekten.

\section{Einleitung}

Emotikons stellen komplexe Zeichen der analogen (z.B. auf Papier), mobilen (z.B. via SMS) und Internetkommunikation (z.B. auf Facebook) dar, die einen emotionalen Zustand ausdrücken und die fehlende mimische Präsenz von Gesprächspartnern kompensieren sollen. Ihr Begriff wurde zum Ende des 20. Jahrhunderts hin in Analogie zum gleichbedeutenden englischen emoticon gebildet, das eine Kontamination der Wörter emotion (,Emotion') und icon (,grafisches Sinnbild') ist. Kombiniert man die Wortbedeutungen, so kommt man der oben genannten Grundfunktion sehr nahe. Bisher wurden Ansätze semiotischer Klassifizierungen und zeichentheoretisch fundierter Strukturdeutungen von Emotikons entwickelt. Gemäß dem Stand der Emotikon-Entwicklung beschränkt sich David Sanderson in seinem "Lexikon der Emotikons“ aus dem Jahr 1997 auf Smileys, deren Form aus dem Zeichensatz der Computertastatur zusammengestellt ist und die mit 
einem 90 Grad nach links geneigten Kopf gelesen werden sollen (vgl. Sanderson 1997). Søren Kjørup stellt sie in einem 2004 veröffentlichten Aufsatz als Unterart der Piktogramme dar und spricht innen zugleich den Bildcharakter ab. Hierbei unberücksichtigt bleiben Emotikons, die auch die Goodman'schen Kriterien für Bildhaftigkeit (kontinuierliche Korrelation, relative Fülle etc.) erfüllen - wie etwa Emotikons der Mangakultur (vgl. Kjørup 2004: 3504). In dem Aufsatz „Die Als-ob-Struktur von Emotikons im WWW und in anderen Medien" (vgl. Wu und Trautsch 2011), der von den Autoren des vorliegenden Textes verfasst wurde, steht im Sinne Hans Vaihingers (1911) die Als-ob-Struktur von Emotikons, d.h. deren Mechanismen zur Erzeugung von zweckmäßigen Einbildungen und Fiktionen im Vordergrund. Hierbei werden in Anlehnung an die Gestentypen nach Ekman (1980) eine Klassifikation der Verwendungsweisen von Emotikons und eine Querklassifikation zwischen den Peirce'schen Objektrelationen Ikon, Index und Symbol und den Kategorien „Zugang zur Emotion“, „Kulturelle Bedingtheit“ und „Entstehungsweise“ aufgestellt. Letztere Klassifikation soll im Folgenden noch einmal hinterfragt und präzisiert werden, da in dieser die Mimik bei Emotikons lediglich den Ikons zugeordnet wird und eventuelle indexikalische oder symbolische Dimensionen außen vor bleiben. Die arbeitspraktische Leitfrage lautet daher: Sind mimische Emotikons per se ikonisch oder entfaltet sich deren Bedeutung vielmehr auf indexikalischer oder symbolischer Ebene? Gemäß dem Thema des vorliegenden Zeitschriftenheftes stehen hierbei - nach einigen theoretischen Grundüberlegungen - schwerpunktmäßig lachende und lächelnde Emotikons im Fokus, wobei die Ergebnisse einer vergleichenden Untersuchung im deutsch- und chinesischsprachigen Raum vorgestellt werden.

\section{Theoretische Grundlagen}

\subsection{Zur Repräsentation von Zeichentypen durch Emotikons}

Emotikons können denselben Zeichentypen wie die ihnen entsprechenden realen Gesichtsausdrücke zugeordnet werden. Auf analoge Weise nehmen Emotikons die Rolle des Signals, des Anzeichens, des Ausdrucks und der Geste im Sinne Posners ein (vgl. Posner 1993 und 1996). Si g n a le sind demnach Zeichen, die bei einem reagierenden System a eine Reaktion $T(a, r)$ auslösen, was mit der Formel $E(f) \rightarrow T(a, r)$ dargestellt wird. Ein Ereignis $f$ (z.B. Donner) - das Signal (Zeichen) - führt zu einem unbewussten Reaktionsprogramm $r$ (z.B. Weghoppeln) durch $a$ (z.B. Kaninchen). Gesichtsausdrücke können Signale sein, insofern sie zu einer Gefühlsansteckung führen, wobei es im Schritt 1 zu einer unbewussten Imitation von Gesichtsausdrücken (z.B. Lächeln) und im Schritt 2 zu einer Stimmungsveränderung gemäß der ursprünglichen Mimik (z.B. Freude) kommt (vgl. Hatfield, Cacioppo und Rapson 1994: 48). Diese Gefühlsansteckung kann auch bei Emotikons verzeichnet werden. Führt ein Ereignis $f$ (Zeichen) 
bei einem reagierenden System a dazu, dass eine bestimmte Proposition $p$ geglaubt wird, spricht man - ganz gleich, ob inr Inhalt der Wirklichkeit entspricht - von einem Anze i che n mit der Formel $E(f) \rightarrow G(a, p)$ (vgl. Posner 1996: 1660ff.). So kann ein plötzliches grollendes Geräusch $f$ auf einer Skipiste für einen Skifahrer a Anzeichen für die Annahme $p$ sein, dass das Abgehen einer Lawine bevorsteht (vgl. Posner 1996: 1660). Gesichtsausdrücke und Emotikons können gleichermaßen Anzeichen für den jeweiligen gemeinten emotionalen Zustand sein: z.B. ;-) für ,glücklich` und :-( für ,unglücklich‘. Im Regelfall dürften sie aber als Ausdrücke zu deuten sein. Beim A u s d r u ck schließt ein reagierendes System a von einem Zeichen $f$ auf die Existenz eines anderen Systems $b$, das sich im Zustand $Z$ befindet: $E(f) \rightarrow G(a, Z(b))$. Auch hier genügt die bloße Annahme des Auftretens von $b$ und $Z(b)$. Weder $Z(b)$ noch $b$ selbst müssen tatsächlich gegeben sein (vgl. Posner 1996: 1661ff.). Als gutes Beispiel für einen Ausdruck kann die durch eine Person a vorgenommene Deutung $G$ von nach unten gezogenen Mundwinkeln und einer nach oben gezogenen Augenbraueninnenseite im Gesichtsausdruck $f$ einer Person $b$ als Zeichen für die momentane Traurigkeit von $Z$ gelten. Dies gilt sowohl für die reale Mimik als auch für komplexere Emotikons. Schließt ein reagierendes System a von einem Ereignis $f$ auf ein mögliches Verhalten $T$ eines anderen Systems $b$ bzw. führt ein Ereignis $f$ dazu, dass a annimmt, dass $b$ intendiert, $g$ zu tun $(T(b, g))$, so liegt nach Posner eine G e s te vor: $G(a, I(b, T(b, g)))$. In Kombination mit einem Emotikon, das eine Bratwurst darstellt, könnte ein Smiley mit einem Ausdruck von Ekel Geste für ein geplantes Vermeidungsverhalten sein.

\subsection{Klassifikation der Arten von Kommunikation mit Emotikons}

Emotikons stellen ein wichtiges Mittel bei der analogen, mobilen und Internetkommunikation dar. Nach dem hier vertretenen Zeichenmodell (vgl. Posner 2010) liegt Kommunikation genau dann vor, wenn an einer S e m i o se die Zeichenbestandteile Sender (z.B. Schulleiter), Adressat (Empfänger des Senders: z.B. Schüler), Z e i c h e n im engen Sinne (z.B. ein strafendes Gespräch), B o t s c h a ft (z.B. ,Du hast mehrfach gegen die Schulordnung verstoßen'), K a n a I (hier: Schallwellen) und Ko n t ext (in diesem Fall: Schule) beteiligt sind. Posners Modell lässt sich auf Zeichenprozesse aller Kanäle und Zeichensysteme anwenden, so auch auf Emotikons. Demnach ließe sich ein Facebook-Chat mit einem lachenden Smiley folgendermaßen visualisieren (vgl. Abb. 1).

Eine Besonderheit der Kommunikation mit Emotikons besteht in der Art und Weise, wie emotionale Zustände repräsentiert werden. Wie die Mimikforschung zeigt, lassen sich Emotionen in realen Kommunikationsformen (faceto-face-Gespräch) nur schwer verbergen oder vortäuschen. Dagegen müssen die Gesichtsausdrücke in Emotikons keine unmittelbare Folge eines emotionalen Zustands sein, da sie bewusst und wohlüberlegt gewählt werden. 


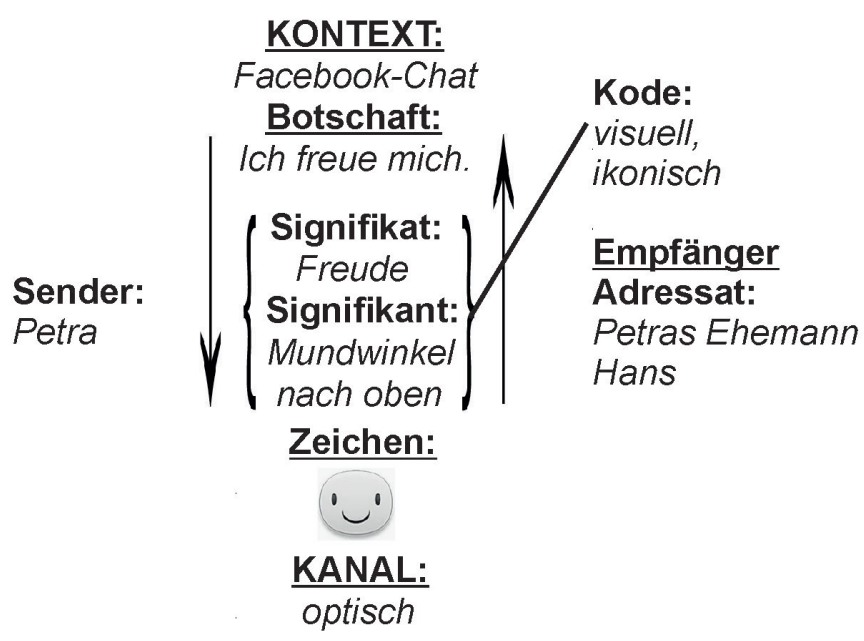

Abb.1: Kommunikation im Chat nach dem Zeichenmodell von Roland Posner (2010: 159).

In Anlehnung an die Gestenklassifikation von Ekman (1980: 98) wurden die kommunikativen Funktionen in Wu und Trautsch (2011) folgendermaBen klassifiziert:

(1) Sprachtextbegleitende Emotikons: Sie treten entweder texträumlich nebeneinander oder textzeitlich nacheinander auf. Dazu gehören:

(a) Bet on ende Emotikons: Sie heben einzelne Wörter hervor. Z.B. „Heute habe ich einen süßen Hund gesehen!“

(b) Unterstreichende Emotikons: Sie heben den ganzen Satz hervor.

Z.B. „Das war wieder einmal ein richtig mieser Tag!

Bei (1a) und (1b) besteht die Hauptfinktion im Wunsch der Vermeidung von Missverständnissen.

(c) Antithetische Emotikons: Es besteht ein konträrer oder kontradiktorischer Gegensatz zwischen dem Sprachtext und dem verwendeten Emotikon. Antithetische Emotikons dienen besonders als Ironiesignale. Benutzer von Emotikons neigen dazu, im Zweifelsfall dem Bild zu glauben.

Z.B. „Heut geht's mir mal wieder super!!! (6) “, wobei der traurige Smiley der Aussage widerspricht und als Ironiesignal gelten kann.

(2) Sprachtextersetzende Emotikons: Es werden lediglich Emotikons texträumlich nebeneinander oder textzeitlich nacheinander verwendet. Hierbei können sich diverse Bedeutungen, Bedeutungskombinationen und kommunikative Absichten ergeben.

So spielt z.B. die Zeichenfolge „, "auf anstrengende körperliche Aktivitäten an. 


\subsection{Problematisierung einer Klassifikation der Objektrelationen von Emotikons}

Die in der Einleitung formulierte arbeitspraktische Leitfrage zielt darauf ab, zu prüfen, ob und, wenn ja, inwiefern die von Wu und Trautsch (2011: 346ff.) aufgestellte Klassifikation der Objektrelationen bzw. des Weltbezugs von Emotikons haltbar und gegebenenfalls zu optimieren ist. Demnach besteht

- die ikonische Relation in einem direkten, kulturunabhängigen und natürlichen Zugang,

- die indexikalische in einem indirekten, weitgehend kulturabhängigen und natürlichen Zugang und

- die symbolische in einem indirekten, kulturabhängigen und künstlichen Zugang zu einer möglichen intendierten Emotion.

Tab.1: Tabelle zur Klassifikation der Emotikons (Wu und Trautsch 2011: 347).

\begin{tabular}{|l|l|l|l|}
\hline Objektrelation & Ikonisch & Indexikalisch & Symbolisch \\
\hline $\begin{array}{l}\text { Zugang zur } \\
\text { Emotion }\end{array}$ & direkt & indirekt & indirekt \\
\hline $\begin{array}{l}\text { Kulturelle Be- } \\
\text { dingtheit }\end{array}$ & kulturunabhängig & $\begin{array}{l}\text { weitgehend kul- } \\
\text { turabhängiug }\end{array}$ & kulturabhängig \\
\hline $\begin{array}{l}\text { Entstehungs- } \\
\text { weise }\end{array}$ & natürlich & natürlich & künstlich \\
\hline
\end{tabular}

Bei der Kategorie „Zugang zur Emotion“ steht die Frage im Vordergrund, ob die Wahrnehmung und Informationsverarbeitung einer Emotion direkt (z.B. Rezeption eines traurigen Gesichtes) oder indirekt (z.B. Gespräch über eine traurige Person) erfolgt. „Kulturelle Bedingtheit“ ist davon abhängig, ob die Semiosen von konventionell kodierten Zeichen geprägt sind oder nicht (vgl. Posner 2003: 42 und 54). Die „Entstehungsweise“ kann entweder als natürlich, das heißt ohne steuerndes Zutun, oder als künstlich, das heißt als beabsichtigt und gesteuert, vom Rezipienten wahrgenommen und empfunden werden. „Ikon“, „Index" und „Symbol“ werden in Anlehnung an Peirce verstanden. Nach ihm weist das Ikon „mit seinem wirklichen oder fiktiven Objekt eine Ähnlichkeit auf“. Ein Index ist dagegen „ein Zeichen, das nicht in einer abbildenden, sondern in einer realen Verbindung mit seinem Objekt steht“, etwa „als Hinweis oder Anzeige". Das Symbol ist sowohl unabhängig von der "Ähnlichkeit“ als auch von "[der] physische[n] Verbindung mit seinem Objekt" und steht somit gewissermaßen dem Ikon und dem Index gegenüber (vgl. Oehler 1979: 14).

Wu und Trautsch (2011) gehen davon aus, dass Smileys als Variante von Emotikons mit Gesichtern Prototypen für ikonische Emotikons sind, da sich deren Verständnis auf die Ähnlichkeit mit realen Gesichtern sowie auf die natürliche Emotionserkennung zurückführen lassen. Diese Einschätzung 
soll ausgehend von der arbeitspraktischen Leitfrage des vorliegenden Textes noch einmal geprüft werden.

\section{Erörterung der arbeitspraktischen Leitfrage}

\subsection{Nach wie vor unumstrittene Argumente für die Ikonizität von Smileys}

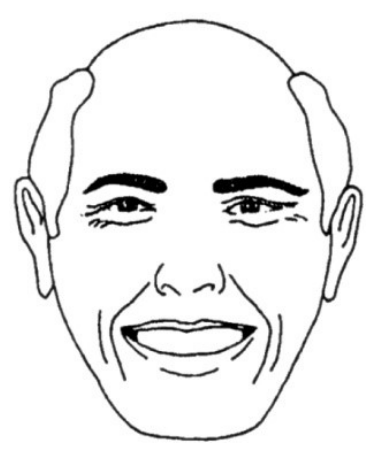

Abb. 2: Mimischer Ausdruck für Freude (Reichardt 2007:7).

In der Tat stellen die Einschätzungen von Emotionsexpressionen und die bei der Darstellung von Basisemotionen beteiligten Muskelbewegungen einen bereits umfassend untersuchten Bereich der Mimikforschung dar. Besonders hervorzuheben sind das von Paul Ekman und Wallace Friesen (1978) entwickelte Kodierungsverfahren Facial Action Coding System und ihre Studien mit indigenen Völkern im australischen Papua-Neuguinea. Neben vielen anderen Untersuchungen, auf die hier im Einzelnen nicht eingegangen werden kann, konnte hierdurch die Kulturunabhängigkeit und Natürlichkeit der Hervorbringung und Deutung des mimischen Ausdrucks der sechs Basisemotionen nachgewiesen werden.

Die Emotion „Freude“ manifestiert sich auf mimischer Ebene durch angehobene Wangen mittels des musculus zygomaticus major (dt.: großer Jochbeinmuskel) und des an der Außenseite zusammengezogenen musculus orbicularis oculi (dt:: Augenringmuskel), der Lachfalten bewirkt (zum Lächeln der Freude vgl. Ekman und Friesen 1982; Frank und Ekman 1993; zum Lachen der Freude vgl. Ruch und Ekman 2001).

Die eben beschriebenen Muskelbewegungen (vgl. Abb. 2) werden von Emotikons aller Komplexitätsstufen ausgedrückt und können daher problemlos der Emotion Freude zugeordnet werden. So beschränken sich die traditionellen aus den USA stammenden Tastaturzeichen-Emotikons auf wesentliche Züge des mimischen Ausdrucks. ,Freude‘ kann hier durch Markierung der hochgezogenen Mundwinkel mit schließenden Klammern wie

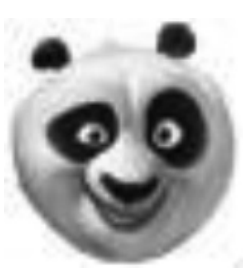

Abb. 3: Kung-Fu-Panda-Emotikon. ${ }^{1}$ etwa durch :) ;-) =) :] :> oder durch Anspielungen auf die Kontraktion des Augenringmuskels z.B. durch $M$ oder $x$ ) ausgedrückt werden. Detailliertere Smileys wie die Kung-Fu-PandaEmotikons versuchen die Muskelform des natürlichen Ausdrucks zu übernehmen, um die Emotion Freude deutlich werden zu lassen.

Auf dieser Ebene kann die bisherige Klassifikation der Objektrelationen von Emotikons also bestätigt werden. 


\subsection{Mögliche Argumente gegen die Ikonizität von Smileys im Zusam- menhang einer Internetbefragung}

Nun stellt sich jedoch die Frage, ob die in 3.1 genannten Bedeutungsspannen wirklich dem tatsächlichen Gebrauch entsprechen und ob es diesbezüglich Unterschiede zwischen geistesgeschichtlich voneinander entfernten Kulturen gibt. Im Zusammenhang des vorliegenden Aufsatzes kam es daher zu einer umfangreichen Internetbefragung im deutsch- und chinesischsprachigen Raum, auf die in Webforen der beiden Länder aufmerksam gemacht wurde. Es nahmen Jugendliche und junge Erwachsene im Alter von 15 bis 32 Jahren aus Deutschland und China teil. Zirka zehn Prozent der deutschen Teilnehmerinnen und Teilnehmer wiesen einen Migrationshintergrund auf, diese hatten aber fast durchgehend deutsche Schulen besucht. Die Emotikon-Auswahl beschränkte sich auf acht Varianten lachender und lächelnder Gelbgesicht-Smileys (siehe Abb. 5), wie sie weltweit in den meisten sozialen Netzwerken gängig sind. Das Fragenformat war von größtmöglicher Offenheit geprägt und spielte auf die intendierten Bedeutungen und die Kontexte an, in denen das jeweilige Smiley verwendet wurde. Die Web-Ergebnisse wurden anschließend in Tabellen gebündelt und ausgewertet, wodurch allgemeine Tendenzen zu erkennen waren. Die chinesischen Einträge und deren Auswertung wurden im Nachhinein ins Deutsche übersetzt. ${ }^{2}$

Insgesamt ließen die deutschen Ergebnisse erkennen, dass Emotikons gerne als für sich sprechend verwendet wurden, wohingegen die chinesischen auf eine bevorzugte Kombination mit Sprache hindeuteten. Was die Objektrelationen angeht, kann bei beiden Kulturen auch eine symbolische Verwendung der Emotikon-Mimik konstatiert werden. Somit könnte der vorschnelle Eindruck entstehen, die Klassifikation in 2.3 (siehe Tab. 1) sei widerlegt.

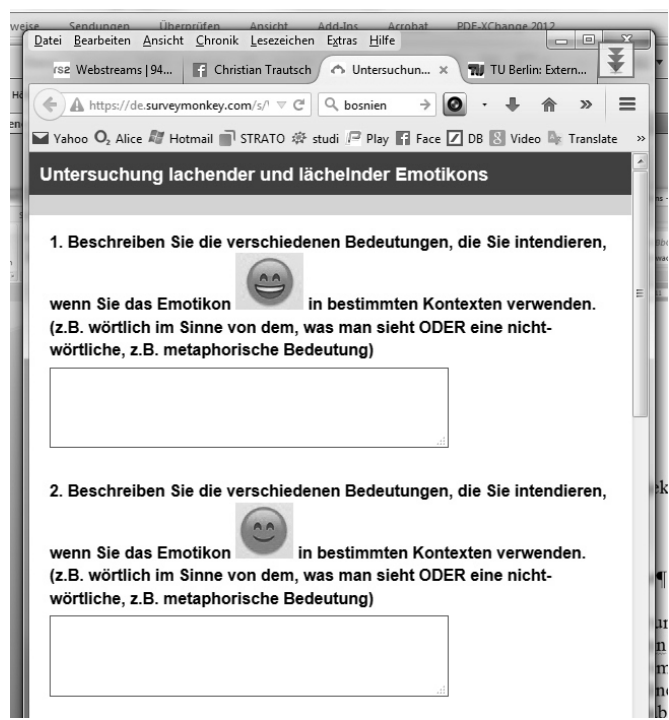

Abb. 4: Befragung von Wu und Trautsch 2015 auf https://de.surveymonkey.com/.

\subsection{Feinanalyse der Untersuchung}

Smiley I und II als Ausdruck: Die Smileys I und II (vgl. Abb. 5) legen sowohl in den deutschen als auch in den chinesischen Einträgen eine Seg- 


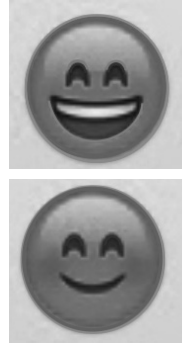

Abb. 5: Smiley I (herzhaftlachend) und II (lächelnd).

mentierung zwischen dem Mund- und dem Augenbereich nahe. Auf deutscher Seite wurde der offene Mund unabhängig vom Kontext einer gesteigerten Intensität der Freude zugewiesen, wohingegen in den chinesischen Ergebnissen schon eine Differenzierung nach sprachlichem Kontext erfolgte. Smiley II stand bei letzteren lediglich für die Emotion Freude, was als ikonisch angesehen werden kann. Smiley I wurde dagegen im Zusammenhang mit Sätzen gebraucht, die einen belustigenden Inhalt hatten. Dies ist zwar insofern symbolisch, als es zur Einbeziehung von Schriftsprache kommt, kann jedoch für sich betrachtet auch als ikonische Intensitätssteigerung durch den offenen Mund gedeutet werden. Hierbei soll die Tatsache ausgeklammert werden, dass die chinesische Schriftsprache selbst einen ikonischen Ursprung besitzt.

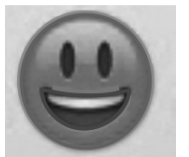

Smiley III als A u s d r u ck: Die ovalen vertikal lang gezogenen Augen in Smiley III wurden auf deutscher Seite mit der gegenüber Smiley I zusätzlichen Bedeutung ,Überraschung' verwendet. Aufgrund der mimischen RepräsentaAbb.6: Smiley III. tion einer weiteren Basisemotion im Sinne Ekmans kann es daher als eindeutig ikonisch klassifiziert werden. In den chinesischen Einträgen dominierten Verwendungsweisen, in denen Freude stark nach außen hin signalisiert werden sollte - eine Deutung, die nicht mehr ohne symbolische Kodierung auskommt.

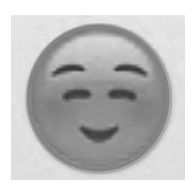

Smiley IV als Anzeichen und Ausdruck: Das lächelnde Smiley IV mit den zusammengekniffenen Augen beinhaltete sowohl auf deutscher als auch chinesischer Seite symbolische Bedeutungszuweisungen. In den deutschen Einträgen überwog die kontextfreie Bedeutung ,intAbb.7: Smiley IV. rovertierter Mensch'. Die Chinesen verwendeten das Zeichen im Zusammenhang mit Sätzen, in denen eine Person alkoholisiert war. Auch wenn introvertierte oder alkoholisierte Menschen unter bestimmten Voraussetzungen eine analoge Mimik aufweisen können, ist hier nicht von rein auf Ähnlichkeit basierenden Deutungen auszugehen. Das Zeichen könnte genauso gut für eine schlafende Person stehen, die einen freudvollen Traum hat.

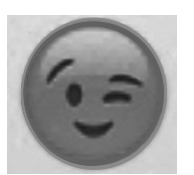

Smiley V als Anzeichen: Das zwinkernde Smiley V wurde in den deutschen und den chinesischen Einträgen gleichermaßen zur Signalisierung von Ironie verwendet. Hier lässt sich kein klarer Zusammenhang mit einer BasiAbb. 8: Smiley V. semotion feststellen, die mit Ironie verbunden werden könnte. Das Emotikon ist daher als Symbol einzustufen.

Smiley VI und Smiley VII als Anze ich en und Ausdruck: Das zwinkernde Smiley VI mit seiner ausgestreckten Zunge wurde in beiden Kulturen als symbolisches Zeichen aufgefasst, das entweder Unernsthaftigkeit ausdrücken oder auf einen Scherz hinweisen sollte. Auch ist eine Segmen- 
tierung zwischen dem Mund- und dem Augenbereich zu verzeichnen. Auf chinesischer Seite zeigte sich diese darin, dass sich aufgrund der ausgestreckten Zunge beim Smiley VII der Bedeutungsschwerpunkt ,Scherz' und ,Unernsthaftigkeit' wiederholte. In den deutschen Ergebnissen war hierbei auch ein ikonischer Zusammenhang zu ermitteln, nach dem die zusammengekniffenen Augen auf das Naserümpfen bei der Basisemotion Ekel anspielten. Wie bereits herausgearbeitet wurde, kann das Aufgreifen mimischer Muster von Basisemotionen als ikonisch angesehen werden.

Smiley VIII als Ausdruck: Beim Smiley VIII überwog die Bedeutung einer Figur, die nachdenklich oder überlegt war, was auf einen ikonischen Zusammenhang mit realen Blickrichtungen schließen lässt.

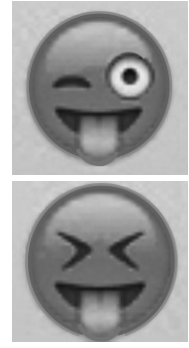

Abb. 9: Smiley VI und VII.

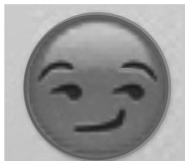

Abb. 10: Smiley VIII.

\section{Beantwortung der arbeitspraktischen Leitfrage}

Unter der arbeitspraktischen Leitfrage, ob mimische Emotikons per se ikonisch sind oder ob sich deren Bedeutung vielmehr auf indexikalischer oder symbolischer Ebene entfaltet, wurden zeichentheoretische Grundüberlegungen mit Bezug auf die psychologische Mimikforschung angestellt und auf eine Untersuchung im chinesischen und deutschen Kulturraum bezogen. Während eindeutig indexikalische Dimensionen nicht ermittelt werden konnten, ließen die Ergebnisse vielmehr auf ein Wechselspiel von Ikonizität und Symbolizität schließen. Erstere überwog hierbei quantitativ, was jedoch nichts daran ändert, dass eine gänzliche Zuordnung mimischer Emotikons zu den Ikons unzulässig ist. Die Klassifikation, nach der Ikons, Indexe und Symbole quer zu „Zugang zur Emotion“, „Kultureller Bedingtheit“ und „Entstehungsweise" unterschieden werden, selbst infrage zu stellen, wäre dennoch zu kurz gegriffen, da sie - wenn auch sicher noch optimierbar - im Kern fundiert ist. Sinnvoller wäre daher die Frage nach der Zuordnung von Emotikons zu den Klassen. Mimische Emotikons müssten demnach in zwei Typen eingeteilt werden: ein Typ, der ohne konventionell kodierte Zeichen auskommt und dessen Bedeutung auf der Grundlage des Ausdrucks von Basisemotionen (z.B. Übernahme einer Kontraktion des Augenringmuskels durch Akzente) oder von Blickverhalten (siehe z.B. Smiley VIII in 3.3) erschlossen werden kann; und ein Typ, dessen Bedeutungsgehalt an konventionell kodierte Zeichen gebunden ist (z.B. das Zunge-Herausstrecken in Smiley VI und VII in 3.3). Erstere könnten problemlos den ikonischen und letztere den symbolischen Emotikons der bisherigen Klassifikation zugeordnet werden. Somit ließe sich die arbeitspraktische Leitfrage mittels einer Synthese auflösen: Mimische Emotikons können abhängig von der Kategorie „Kulturelle Bedingtheit" (siehe 2.3) entweder ikonisch oder symbolisch sein. Indexikalische Varianten sollen bis auf weitere Untersuchungen als möglich gelten. 


\section{Anmerkungen}

1 Die Kung-Fu-Panda-Smileys wurden am 03.06.2008 auf der Website www.kungfupanda.com abgerufen. Diese ist nicht mehr online.

2 Die chinesische Variante der Studie und die Übersetzungsarbeiten wurden von Yixin Wu durchgeführt. Christian Trautsch wertete die deutsche Untersuchung aus.

\section{Literatur}

Ekman, Paul (1980), „Three classes of nonverbal behavior“. In: Walburga von RafflerEngel (Hrsg.), Aspects of Nonverbal Communication. Lisse: Swets and Zeitlinger: 89-102.

Ekman, Paul und Wallace V. Friesen (1978), The Facial Action Coding System (FACS): A Technique for the Measurement of Facial Action. Palo Alto: Consulting Psychologists Press.

Ekman, Paul und Wallace V. Friesen (1982), „Felt, False, and Miserable Smiles“. Journal of Nonverbal Behavior 6, 4: 238-252.

Frank, Mark G. und Paul Ekman (1993), „Not all smiles are created equal: the differences between enjoyment and nonenjoyment smiles“. Humor. International Journal of Humor Research 6, 1: 9-26.

Hatfield, Elaine, John T. Cacioppo und Richard L. Rapson (1994), Emotional Contagion. Cambridge: Cambridge University Press.

Kjørup, Søren (2004), „Pictograms“. In: Roland Posner, Klaus Robering und Thomas A. Sebeok (Hrsg.), Semiotik. Ein Handbuch zu den zeichentheoretischen Grundlagen von Natur und Kultur. Bd. 4. Berlin: De Gruyter: 3504-3510.

Oehler, Klaus (1979), „Idee und Grundriß der Peirceschen Semiotik“. Zeitschrift für Semiotik 1, 1: 9-22.

Posner, Roland (1993), „Believing, Causing, Intending: The Basis for a Hierarchy of Sign Concepts in the Reconstruction of Communication". In: René J. Jorna, Barend van Heusden und Roland Posner (Hrsg.), Signs, Search, and Communication. Semiotic Aspects of Artificial Intelligence. Berlin und New York: De Gruyter: 215-270.

Posner, Roland (1996), „Sprachphilosophie und Semiotik“. In: Marcelo Dascal, Dietfried Gerhardus, Kuno Lorenz und Georg Meggle (Hrsg.), Sprachphilosophie: Ein internationales Handbuch zeitgenössischer Forschung. Berlin und New York: De Gruyter: 1658-1685.

Posner, Roland (2003), „Kultursemiotik“. In: Ansgar Nünning und Vera Nünning (Hrsg.), Konzepte der Kulturwissenschaften. Stuttgart und Weimar: Metzler: 39-71.

Posner, Roland (2010), „Die Wahrnehmung von Bildern als Zeichenprozess“. In: Dieter Maurer und Claudia Riboni (Hrsg.), Bild und Bildgenese. Stuttgart: Reimer: 139-183.

Reichardt, Nils (2007), Mimik und Gestik bei virtuellen Charakteren. URL: https://wwwvs.informatik.uni-ulm.de/teach/ss07/3d/ausarbeitung/Nils\%20Reichardt\%20-\%20 Mimik\%20und\%20Gestik.pdf. [Letzter Zugriff am 13.05.2015].

Ruch, Willibald und Paul Ekman (2001), „The expressive Pattern of Laughter“. In: Alfred W. Kaszniak (Hrsg.), Emotion, Qualia, and Consciousness. Tokyo: World Scientific Publisher: 426-433. 
Sanderson, David (1997), „Lexikon der Emotikons“. Zeitschrift für Semiotik 19, 3: 307315.

Vaihinger, Hans (1911), Die Philosophie des als ob: System der theoretischen, praktischen und religiösen Fiktionen der Menschheit auf Grund eines idealistischen Positivismus. Mit einem Anhang über Kant. Berlin: Reuther \& Reichard.

Wu, Yixin und Christian Trautsch (2011), „Die Als-ob-Struktur von Emotikons im WWW und in anderen Medien“. Kodikas/Code. Ars Semeiotica 34, 3-4: 343-356.

M.A. Yixin Wu und StR Christian Trautsch

Technische Universität Berlin

Arbeitsstelle für Semiotik, FH 448

Fraunhoferstraße 33-36

D-10587 Berlin

E-Mail: trautsch@semiotik-forum.de 